\title{
La evaluación de competencias de escritura a través de portafolios en el nivel Bachillerato'
}

\author{
FRANCISCO SAMUEL MENDOZA MOREIRA \\ JIMMY ANDRÉS VILLEGAS \\ Universidad Laica Eloy Alfaro de Manabí \\ Ecuador \\ samuel.mendoza@uleam.edu.ec \\ jimandres.villegas@gmail.com
}

The assessment of writing skills through Portfolios in High School Level

(Recibido: O7-I2-20I5; aceptado: II-O2-2OI6)

Resumen. En la sociedad del conocimiento, hablar de formación para la comunicación es abordar el campo de las competencias comunicativas que se expresan de acuerdo a los diferentes canales de expresión humana y los códigos propios del lenguaje. El currículo ecuatoriano pretendiendo mantener sincronía con el mundo presenta una estructura curricular que pretende el desarrollo de estas competencias en los estudiantes aspirantes del bachillerato, desencadenando entre otras de sus situaciones problémicas la evaluación integral de las mismas.

A través del estudio realizado se provee de experiencias y técnicas que permitan la evaluación eficaz de las mismas, convirtiendo al portafolio en una experiencia de transformación del sujeto que aprende a través de la colección de evidencias que se presenta en el desarrollo del currículo de formación en base a la experiencia realizada en la Unidad Educativa Juan Montalvo de la Facultad Ciencias de la Educación.

Palabras clave: lengua escrita; evaluación pedagógica; entorno educacional; proceso de aprendizaje.
Abstract. In a knowledge based society, to talk about communication training is to address the field of communication skills that are expressed according to different channels of human expression and codes of language. The Ecuadorian curriculum, in an effort to be up to date with the world, presents a curricular structure that aims to develop these skills in aspiring high school students. This triggers, among other problematic situations, a comprehensive assessment of this curriculum.

In this study experiences and techniques are provided that enable effective assessment, making the portfolio a transformational experience of the learner through the collection of evidence presented in curriculum development training, based on the experience in the School Unidad Educativa "Juan Montalvo" of the Department of Education in Ecuador.

Keywords: written language; student study team; educational environment; learning process.

\footnotetext{
${ }^{\text {I }}$ Para citar este artículo: Mendoza Moreira, Francisco Samuel y Villegas Barreiro, Jimmy Andrés (20I7). La evaluación de competencias de escritura a través de portafolios en el nivel Bachillerato. Alabe 15. [www.revistaalabe.com]

DOI: IO.I5645/Alabe2OI7.I5.9
} 


\section{Introducción}

La práctica innovadora y de construcción de nuevos espacios de aprendizaje, permite involucrar en la formación de bachilleres nuevas prácticas vinculadas con los horizontes epistemológicos que orientan la formación secundaria. Es el caso de la unidad educativa "Juan Montalvo", centro de experimentación y práctica docente de la facultad Ciencias de la Educación de la Universidad Laica Eloy Alfaro de Manabí, en que los estudiantes de las carreras y los docentes investigadores generan nuevas formas de aprender en la era de la complejidad.

Para evaluar la competencia comunicativa, a nivel de escritura, se ha desarrollado un marco teórico que permite la concepción epistemológica del objeto de evaluación, permitiendo así organizar acciones transversales en el currículo oficial que aborden desde los contenidos programados el desarrollo permanente de la escritura como forma de pensamiento y de organización intelectual.

Con base a esta nueva concepción de la escritura, se genera un marco operativo que permite direccionar las acciones evaluativas en un proceso dialógico de evaluación a través del portafolio, que concebido de manera cierta es una herramienta metacognitiva que asegura la consolidación de la competencia escritural desde la historicidad de los productos de escritura generados desde los objetos de conocimiento del currículo formal.

Se presenta en este artículo, el marco referencial y operativo de los procesos de evaluación de la competencia de escritura a través del uso de un portafolio que se alimenta de las diferentes experiencias de los sujetos de aprendizaje en el nivel bachillerato.

\section{La competencia escritural: Una mirada desde el bachillerato}

Los horizontes epistemológicos que organizan el conocimiento y explican la capacidad humana de poder escribir de manera coherente y comprensible para los demás, coincide teóricamente en el abordaje de la competencia comunicativa como el fin máximo perseguido en los procesos de escritura, sea ésta con fines recreacionales o de comunicación académica en todos los niveles.

Hymes, citado por Pilleux (200I:35) sostiene que:

... la competencia comunicativa se ha de entender como un conjunto de habilidades y conocimientos que permiten que los hablantes de una comunidad lingüística puedan entenderse. En otras palabras, es nuestra capacidad de interpretar y usar apropiadamente el significado social de las variedades lingüísticas, desde cualquier circunstancia, en relación con las funciones y variedades de la lengua y con las suposiciones culturales en la situación de comunicación. 
Asumiendo la postura de Hymes, al abordar las variedades lingüísticas desde cualquier circunstancia, es necesario analizar la estructura de la misma como un todo referido desde la combinación apropiada de los elementos lingüísticos y pragmáticos que hacen posible la comunicación en diferentes canales y sistema de códigos, considerando que nos comunicamos desde el inicio de nuestros días hasta que dejamos de respirar.

De esta manera, al entender la competencia comunicativa como un constructo lingüístico propio de los seres humanos, resultado de la evolución de diversas formas de expresión y de organización de signos, significados y significantes, que permiten la comprensión de los mensajes de manera apropiada considerando una serie de elementos que no se pretende abordar en este artículo, sino enlistar para reconocer el aporte de cada uno de ellos a la producción escrita como manifestación de competencias cognitivas.

Estas organizaciones estructurantes de la competencia comunicativa, responden al criterio lingüístico y pragmático de la misma, permitiendo entonces entender la relación existente entre lo gramatical y lo textual en una dimensión comprensiva del lenguaje y desde la dimensión pragmática, las funciones locutivas y sociolingüísticas que permiten su contextualización. (Véase ilustración $\mathrm{N}^{\circ} \mathrm{I}$ )

Ilustración I: Definición estructural de la competencia comunicativa

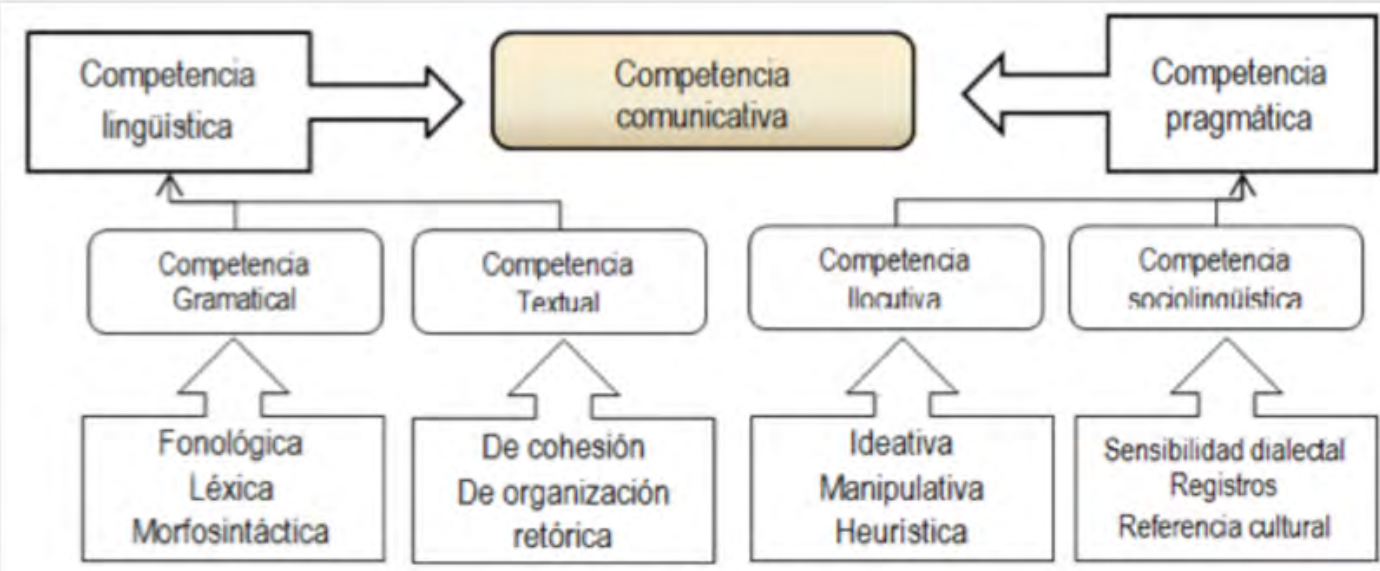

Fuente: Mendoza, et.al (2014:2I) Estrategias para la sensibilización y atención en el aprendizaje de la lengua.

Para el desarrollo de la competencia comunicativa, se debe asumir que los elementos constitutivos del lenguaje se aprenden y desarrollan desde la práctica misma del lenguaje, "a hablar se aprende hablando, a leer, leyendo y a escribir, escribiendo" Mendoza (2006); por tanto el currículo de Lengua y Literatura vigente en Ecuador, debe concatenar el aprendizaje de la lengua con su práctica permanente, de manera que los componentes curriculares se orienten de manera clara hacia la comunicación. (Véase ilustración 2) 
Ilustración 2: Estructura del currículo de Lengua y Literatura en bachillerato

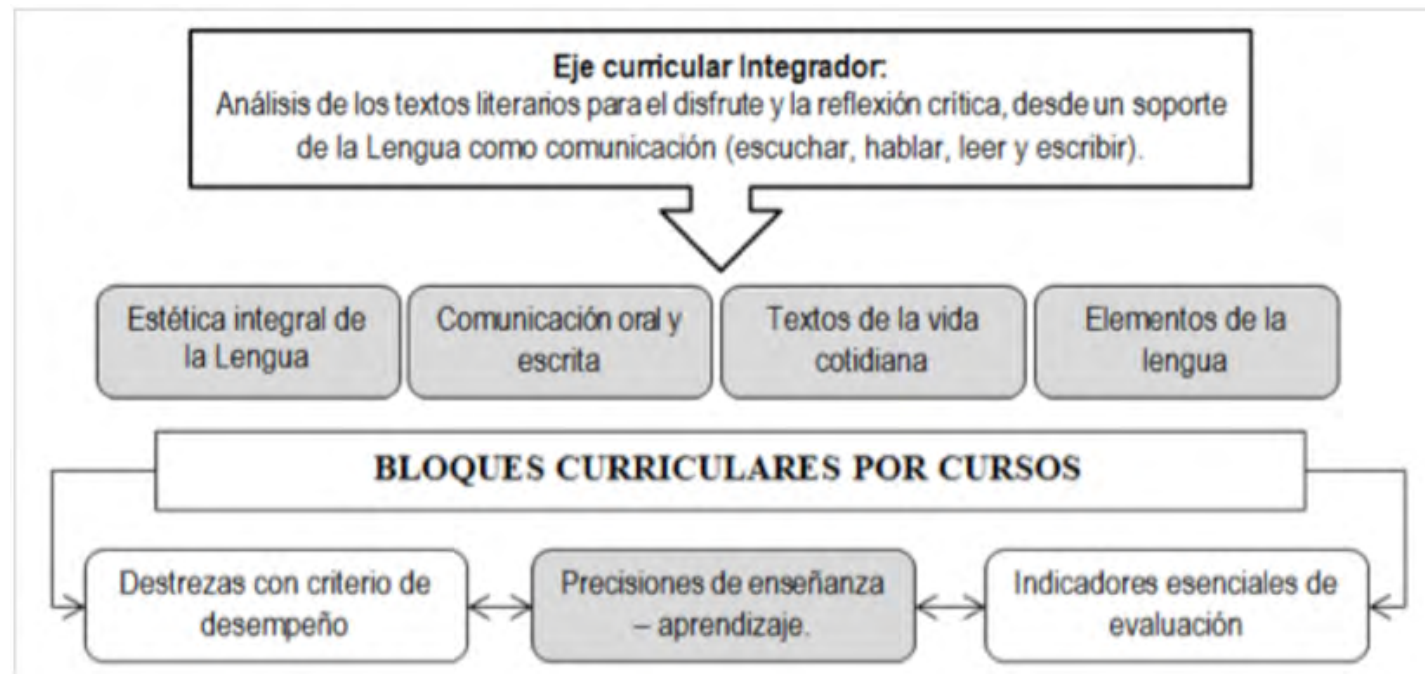

Fuente: Mendoza, et.al (2014:22) Estrategias para la sensibilización y atención en el aprendizaje de la lengua

Desde esta definición conceptual y curricular de los elementos que se conectan para direccionar el aprendizaje de lengua y literatura en el bachillerato surge entonces la necesidad de identificar los elementos propios de la competencia comunicativa por vía escrita, que deben ser construidos y evaluados en el proceso de aprendizaje de los sujetos aprendices, generando desde este espacio una propuesta organizada de las habilidades que se requieren para levantar este constructo.

Conceptualmente, se asume a la competencia lingüística como "un conjunto organizado de conocimientos formado por varios componentes: el componente fonológico, el componente morfológico, el componente sintáctico, el componente semántico y el componente léxico (Belinchón, Igoay Rivière, I994: 261-262)" citados por Trujillo (2OIO:38); esta definición permite entender entonces que la competencia comunicativa es una construcción colectiva de diferentes elementos que unidos entre sí permiten la expresión escrita del pensamiento.

Para llegar al objeto de estudio de este artículo, se requiere entonces señalar una serie de indicadores que en estudios realizados por Florez y otros (2005:35), generan una secuencia de indicadores progresivos que se construyen de manera sistemática desde la Educación Básica hasta el Bachillerato y que se perfecciona en la formación profesional. (Véase Tabla I)

Para poder construir un proceso de evaluación contextualizado, se genera una relación horizontal entre los estándares de calidad de Desempeño Estudiantil (Ministerio de Educación, 2OI2) con referencia al aprendizaje del lenguaje en los diversos niveles del sistema educativo, permitiendo así organizar la metodología instrumentada de evaluación a través de portafolios. 
Tabla I: Relación de estándares nacionales con las categorías de la competencia de escritura

\begin{tabular}{|c|c|c|}
\hline $\begin{array}{l}\text { Categoria } \\
\text { subeategoría }\end{array}$ & Indicadores & Estándares nacionales \\
\hline $\begin{array}{l}\text { Coherencia local- } \\
\text { concordancia }\end{array}$ & $\begin{array}{l}\text { Producir al menos un enun- } \\
\text { ciado y establecer concor- } \\
\text { dancia entre el sujeto y el ver- } \\
\text { bo al interior del mismo. }\end{array}$ & \multirow{3}{*}{$\begin{array}{l}\text { - Produce textos sencillos relacionados } \\
\text { con sus intereses. } \\
\text { - Produce textos escritos literarios y no } \\
\text { literarios de poca extensión, para narrar } \\
\text { y describir. } \\
\text { - Produce textos escritos, literarios y no } \\
\text { literarios, para exponer o informar. } \\
\text { - Produce textos escritos literarios y no } \\
\text { literarios de carácter argumentativo. Es- } \\
\text { tablece metas y objetivos personales para } \\
\text { la producción de sus textos. } \\
\text { - Produce varios tipos de textos litera- } \\
\text { rios: composiciones y textos no literarios } \\
\text { de carácter argumentativo y expositivo². }\end{array}$} \\
\hline $\begin{array}{l}\text { Coherencia local- } \\
\text { segmentación }\end{array}$ & $\begin{array}{l}\text { Segmentar debidamente } \\
\text { enunciados mediante algún } \\
\text { recurso explícito como espa- } \\
\text { cios o signos de puntuación. }\end{array}$ & \\
\hline $\begin{array}{l}\text { Coherencia lineal- } \\
\text { progresión temática }\end{array}$ & $\begin{array}{l}\text { Producir más de un enuncia- } \\
\text { do y seguir un hilo temático a } \\
\text { lo largo del texto. }\end{array}$ & \\
\hline $\begin{array}{l}\text { Coherencia global - conec- } \\
\text { tores con función }\end{array}$ & $\begin{array}{l}\text { Establecer relación explícita } \\
\text { entre los enunciados a través } \\
\text { del uso de algún conector o } \\
\text { frase conectiva. }\end{array}$ & \multirow{2}{*}{$\begin{array}{l}\text { - Utiliza imágenes que expresen ideas en se- } \\
\text { cuencia, escribe con sus propios códigos y } \\
\text { algunas palabras familiares. } \\
\text { - Organiza sus ideas sobre un tema especí- } \\
\text { fico con coherencia. Emplea un vocabulario } \\
\text { coloquial o formal, de acuerdo con la situa- } \\
\text { ción o tema. Aplica las normas ortográficas } \\
\text { en oraciones simples; utiliza correctamente } \\
\text { las mayúsculas, la coma y el punto. } \\
\text { - Organiza ideas sobre un tema central y } \\
\text { considera la estructura de un texto. Utiliza } \\
\text { las partes de un párrafo introductorio y con- } \\
\text { clusivo. } \\
\text { - Desarrolla ideas con coherencia y cohesión } \\
\text { sobre un tema central y considera la estruc- } \\
\text { tura de un texto. } \\
\text { - Utiliza las propiedades textuales: estruc- } \\
\text { tura, coherencia, cohesión y adecuación. } \\
\text { Escribe resúmenes de textos, ensayos argu- } \\
\text { mentativos, expositivos, y de análisis y crítica } \\
\text { literaria. }\end{array}$} \\
\hline $\begin{array}{l}\text { Coherencia global - signos } \\
\text { de puntuación }\end{array}$ & $\begin{array}{l}\text { Evidenciar relaciones entre } \\
\text { enunciados mediante el uso } \\
\text { de signos de puntuación. }\end{array}$ & \\
\hline
\end{tabular}

${ }^{2}$ El documento de estándares de aprendizaje, no señala con precisión los temas ni el nivel de complejidad del texto, esto queda en criterio del evaluador y del desarrollo curricular según el nivel que aglutina el campo de aplicación.

${ }^{3}$ Se toma como referencia el contenido propuesto en los textos oficiales del Ministerio de Educación, los contenidos planificados con base en el Currículo Oficial del Bachillerato General Unificado. 
Siguiendo el pensamiento de Desinano y Avendaño (2006:I8), "el aprendizaje de la escritura, demanda una serie de procesos conectados entre sí que permiten que el pensamiento se haga expreso mediante el uso de las palabras y el lenguaje como canal de explicitación de los procesos conscientes e inconscientes de aprendizaje"; esto implica entonces que los procesos de escritura no son un tema que se aborda en el aula de clases, sino una práctica permanente, regulada y controlada de los sujetos que aprenden a escribir.

\section{La evaluación de la competencia de escritura a nivel Bachillerato}

La evaluación es un proceso que permite el levantamiento permanente de información que parte del aprendizaje de los sujetos involucrados en el acto educativo, la misma que debe ser juzgada e interpretada para con base en ello poder tomar acciones que permitan la construcción significativa de habilidades comunicativas y expresivas que respondan a las necesidades del contexto en que se aprende.

Vista así la evaluación, no es un proceso que se realice únicamente al inicio y al final de un proceso, sino que se mantiene activo en cada etapa de aprendizaje permitiendo así identificar los elementos que se consolidan y aquellos que requieren una forma diferente de abordaje.

Si se considera lo que señala Chomsky (2009:48):

... "la competencia lingüística es la capacidad que tiene todo ser humano de manera innata de poder hablar y crear mensajes que nunca antes había oído. Esta competencia se centra en las operaciones gramaticales que tiene interiorizadas el individuo y se activan según se desarrolle su capacidad coloquial. Es decir, el lenguaje nace desde dentro del individuo y no desde lo social como sostenía Saussure. El maestro lo que tiene que hacer es desarrollar esta competencia lingüística en el alumno haciéndole que hable y enseñándole vocabulario y no solamente gramática. La competencia lingüística se hace realidad a través de reglas generativas que se relacionan con la gramática que es saber organizarse y estructurarse”

Desde esta concepción del objeto de estudio y del mecanismo de transformación, que en este caso es la evaluación; la competencia de escritura no puede evaluarse como tradicionalmente se han venido explorando los aprendizajes, basados en pruebas de preguntas y respuestas sobre el significado de los elementos lingüísticos, sino que se debe recurrir a proceso de evaluación auténtica que permitan la manifestación de los aprendizajes relevantes, que de acuerdo con Pérez (2012:96), son "aquellos que mueven a los sujetos a buscar nuevas formas de aplicarlo y de utilizarlo"

Álvarez (2OI2:I38), sostiene que, "el carácter auténtico de la evaluación queda definido por su vínculo con el mundo real, con la vida cotidiana”; por tanto para que la evaluación de la escritura sea auténtica y real, deberá atender los intereses propios de los sujetos, 
es decir, de aquello que en su mundo es trascendente e importante, aunque para el profesor no lo sea; y desde las demandas propias del contexto en que se desarrolla el currículo, para abordar los elementos fundamentales de la oferta formativa y finalmente del entorno en que se construye el aprendizaje.

En este contexto, el aprendizaje del lenguaje, es el medio con que desarrollan destrezas que garantizan la inserción del sujeto que aprende en la sociedad. El lenguaje se convierte en el principal instrumento de inserción social y de construcción de los colectivos imaginarios que se dan en contextos propios de desarrollo: relaciones de amistad, participación en actividades comunitarias, proceso educativo propiamente dicho, relaciones familiares e inclusive aborda niveles mayores de intimidad, que sin desconocer los medios y códigos disponibles en la actualidad, se mantiene el uso del lenguaje como la herramienta fundamental de socialización.

Este tipo de evaluación que se propone para el abordaje de la competencia de escritura, está basada en desafíos cognitivos que aseguran la manifestación máxima de las capacidades de los sujetos de aprendizaje, principios de autorregulación y metacognición que favorecen no solamente a los procesos de escritura sino también a los de construcción conceptual e instrumental del currículo de formación y a una serie de experiencias transferibles que permitan desde la acción, aprender consigo mismo, para sí y con los demás, en los contextos que anteriormente se señalaron, siendo estos modificables de acuerdo al registro lingüístico y al nivel de comunicación en que se desarrolla el acto de habla.

Es entonces la evaluación, la que permite la consolidación de los procesos metacognitivos que implican el acto de escribir, haciendo conciencia de la relación que estos tienen con los indicadores (tabla I) de la competencia de escritura, mismos que se describen a continuación: 
Tabla 2: Procesos y habilidades metacognitivas de la escritura

\begin{tabular}{|l|l|}
\hline \multicolumn{1}{|c|}{ Procesos } & \multicolumn{1}{|c|}{ Habilidlades metacognitivas } \\
\hline Planeación & $\begin{array}{l}\text { - Se cuestiona acerca de las posibles opciones que permiten cum- } \\
\text { plir con las demandas impuestas por la tarea. } \\
\text { - Plantea al menos un objetivo específico al iniciar la tarea. } \\
\text { - Reconoce cómo afectan los conocimientos que posee el desarro- } \\
\text { llo de la tarea. } \\
\text { - Identifica elementos y/o estrategias que le permiten llevar a cabo } \\
\text { la tarea. }\end{array}$ \\
\hline Autorregulación & $\begin{array}{l}\text { - Da cuenta de la permanencia del objetivo durante la tarea. } \\
\text { - Utiliza y/o da cuenta de por lo menos una estrategia específica } \\
\text { contrastándola con el objetivo para el éxito de la tarea. } \\
\text { - Identifica falencias o fortalezas conceptuales específicas propias } \\
\text { o de la audiencia que influyen en el comportamiento del objetivo. } \\
\text { - Aplica y da cuenta de estrategias específicas para desarrollar con } \\
\text { éxito la tarea. } \\
\text { - Evoca información necesaria para llevar a cabo la tarea. } \\
\text { - Identifica y corrige a nivel de contenido elementos del escrito y } \\
\text { estrategias utilizadas para hacerlo más comprensible. }\end{array}$ \\
\hline Evaluación & $\begin{array}{l}\text { - Da cuenta de los conocimientos específicos que brida el texto a } \\
\text { la audiencia. } \\
\text { - Identifica elementos del escrito referentes al contenido del mis- } \\
\text { mo, pensando en la audiencia, } \\
\text { - Argumenta por qué permanece el objetivo al finalizar la tarea. }\end{array}$ \\
\hline
\end{tabular}

Fuente: Habilidades metalingüísticas, operaciones metacognitivas y su relación con los niveles de competencia en lectura y escritura: un estudio exploratorio (Florez, Torado, Arévalo, Mesa, Modragon, \& Pérez, 2005)

De lo anteriormente señalado, se puede inferir que la evaluación es un acto de aprendizaje que transforma a las personas, permitiendo su vinculación directa con el objeto de conocimiento (el lenguaje), relación que se construye en permanente diálogo entre quien escribe y lo que escribe, por tanto, la evaluación centrará su interés en verificar las condiciones que el sujeto debe manifestar en la producción de sus escritos. 
Partiendo del sentido evaluativo que se propone en este artículo, es necesario considerar los indicadores de realización de la competencia comunicativa, los estándares nacionales de evaluación de la producción de textos (Ministerio de Educación, 20I2) y las herramientas de conocimiento que van a permitir a partir de la organización del aprendizaje, el logro de estos desafíos comunicativos en los adolescentes, para lo que de acuerdo con Tenbrick (2006:95), el primer momento para evaluar es "describir la información necesaria", esto definir el objeto de evaluación y los elementos que lo construyen para la elaboración de los procesos vinculados con el aprendizaje de los estudiantes.

Los objetos de evaluación basados en la competencia comunicativa escrita, se describen considerando lo propuesto por Tenbrick (2006:98), en cuanto a la metodología para el planeamiento de la evaluación; se orienta desde la construcción epistemológica de procesos comunicativos pragmáticos, es decir, aprender a comunicarse a través de la comunicación; que defienden Niño y Pachon (2009:26); en una propuesta coherente con el contexto de los usuarios de la lengua y las demandas establecidas en el Currículo Oficial de Lengua y Literatura.

A continuación, se presenta la metodología utilizada para la construcción de portafolio de evaluación desde el objeto evaluable de la competencia de escritura: 
Tabla 3: Planificación del objeto evaluable desde la competencia de escritura en el bachillerato

\begin{tabular}{|c|c|c|c|c|}
\hline \multirow{2}{*}{$\begin{array}{l}\text { Objeto de } \\
\text { evaluación }\end{array}$} & \multirow{2}{*}{$\begin{array}{l}\text { Indicadores } \\
\text { de } \\
\text { competencia }\end{array}$} & \multicolumn{2}{|c|}{ Actividades adscritas } & \multirow{2}{*}{$\begin{array}{l}\text { Tarea evaluable } \\
\text { (evidencia } \\
\text { de aprendivaje) }\end{array}$} \\
\hline & & Asistidas por el docente & $\begin{array}{l}\text { Orientadas a la } \\
\text { metacognición }\end{array}$ & \\
\hline $\begin{array}{l}\text { Produce varios } \\
\text { tipos de textos } \\
\text { literarios: com- } \\
\text { posiciones y } \\
\text { textos no litera- } \\
\text { rios de carácter } \\
\text { argumentativo y } \\
\text { expositivo }^{4} \text {. }\end{array}$ & $\begin{array}{l}\text { S e } g \text { m e n t a r } \\
\text { debidamente } \\
\text { enunciados me- } \\
\text { diante algún re- } \\
\text { curso explícito } \\
\text { como espacios } \\
\text { o signos de pun- } \\
\text { tuación. }\end{array}$ & $\begin{array}{l}\text { - Estratificación de las ideas. } \\
\text { - Secuenciación de las ideas } \\
\text { para escribir. } \\
\text { - Organización de las ideas } \\
\text { para escribir. } \\
\text { - Generación de oraciones, } \\
\text { párrafos y renglones con base } \\
\text { en las ideas. } \\
\text { - Revisión por terceros del do- } \\
\text { cumento generado. } \\
\text { - Ajustes basados en las obser- } \\
\text { vaciones realizadas. }\end{array}$ & $\begin{array}{l}\text { - Plantea al menos un ob- } \\
\text { jetivo específico al iniciar } \\
\text { la tarea. } \\
\text { - Da cuenta de la perma- } \\
\text { nencia del objetivo du- } \\
\text { rante la tarea. } \\
\text { - Argumenta por qué } \\
\text { permanece el objetivo al } \\
\text { finalizar la tarea. }\end{array}$ & $\begin{array}{l}\text { Narración de sucesos, } \\
\text { experiencias, emociones } \\
\text { y situaciones a través de } \\
\text { la escritura, haciendo co- } \\
\text { nocer su punto de vista } \\
\text { o presentando de forma } \\
\text { ordenada lo acontecido. }\end{array}$ \\
\hline $\begin{array}{l}\text { Escribe resú- } \\
\text { menes de tex- } \\
\text { tos, ensayos } \\
\text { argumentativos, } \\
\text { expositivos, yde } \\
\text { análisis y crítica } \\
\text { literaria }{ }^{5} \text {. }\end{array}$ & $\begin{array}{l}\text { Establecer re- } \\
\text { lación explícita } \\
\text { entre los enun- } \\
\text { ciados a través } \\
\text { del uso de algún } \\
\text { conector o frase } \\
\text { conectiva. }\end{array}$ & $\begin{array}{l}\text { - Acercamiento al texto litera- } \\
\text { rio (de acuerdo a la prescrip- } \\
\text { ción curricular) } \\
\text { - Análisis de los elementos del } \\
\text { texto (personajes, escenarios, } \\
\text { escenas, consecuencias y de- } \\
\text { rivaciones lógicas) } \\
\text { - Generación de las ideas para } \\
\text { escribir } \\
\text { - Producción del primer bo- } \\
\text { rrador. } \\
\text { - Evaluación en pares delo ge- } \\
\text { nerado. } \\
\text { - Ajustes y precisiones con } \\
\text { base en lo evaluado. }\end{array}$ & $\begin{array}{l}\text { - Identifica elementos } \\
\text { y/o estrategias que le } \\
\text { permite llevar a cabo la } \\
\text { tarea. } \\
\text { - Utilizay/o da cuenta de } \\
\text { por lo menos una estra- } \\
\text { tegia específica contras- } \\
\text { tándola con el objetivo } \\
\text { para el éxito de la tarea. } \\
\text { - Aplica y da cuenta de } \\
\text { estrategias específicas } \\
\text { para desarrollar con éxi- } \\
\text { to la tarea. } \\
\text { - Identifica elementos } \\
\text { del escrito referentes al } \\
\text { contenido del mismo, } \\
\text { pensando en la audien- } \\
\text { cia. }\end{array}$ & $\begin{array}{l}\text { Producción de un texto } \\
\text { corto de hasta } 5 \text { oo pala- } \\
\text { bras en base al asunto cu- } \\
\text { rricular abordado (temas } \\
\text { literarios) }\end{array}$ \\
\hline
\end{tabular}

\footnotetext{
${ }^{4}$ Se hace necesario aclarar, que estos textos, con carácter argumentativo y expositivo, están basados en las necesidades de las otras asignaturas con relación a la producción de los estudiantes por medios escritos.

5 Es necesario aclarar que estos textos se vinculan con la producción literaria en estudio durante el curso escolar: Producción hispanoamericana.
} 


\begin{tabular}{|c|c|c|c|c|}
\hline \multirow{2}{*}{$\begin{array}{l}\text { Objeto de } \\
\text { evaluación }\end{array}$} & \multirow{2}{*}{$\begin{array}{l}\text { Indicadores } \\
\text { de } \\
\text { competencia }\end{array}$} & \multicolumn{2}{|c|}{ Actividades adscritas } & \multirow{2}{*}{$\begin{array}{l}\text { Tarea evaluable } \\
\text { (evidencia } \\
\text { de aprendivaje) }\end{array}$} \\
\hline & & Asistidas por el docente & $\begin{array}{l}\text { Orientadas a la } \\
\text { metacognición }\end{array}$ & \\
\hline $\begin{array}{l}\text { Escribe sus } \\
\text { ideas; opina } \\
\text { y argumenta } \\
\text { sobre diversas } \\
\text { situaciones } \\
\text { sociales de su } \\
\text { entorno y con } \\
\text { propósitos co- } \\
\text { municativos es- } \\
\text { pecíficos. }\end{array}$ & $\begin{array}{l}\text { Responder re- } \\
\text { querimientos } \\
\text { pragmáticos de } \\
\text { la situación de } \\
\text { comunicación. }\end{array}$ & $\begin{array}{l}\text { - Participación en espaciosco- } \\
\text { municativos. } \\
\text { - Identificación del objeto de } \\
\text { escritura. } \\
\text { - Generación de ideas para } \\
\text { escribir. } \\
\text { - Organización del documen- } \\
\text { to. } \\
\text { - Evaluación colectiva de la } \\
\text { producción. } \\
\text { - Ajustes y precisiones basa- } \\
\text { das en la evaluación del escri- } \\
\text { to. }\end{array}$ & $\begin{array}{l}\text { - Reconoce cómo afectan } \\
\text { los conocimientos que } \\
\text { posee el desarrollo de la } \\
\text { tarea. } \\
\text { - Identifica falencias o } \\
\text { fortalezas conceptuales } \\
\text { específicas propias o de } \\
\text { la audiencia que influyen } \\
\text { en el comportamiento } \\
\text { del objetivo. } \\
\text { - Da cuenta de los co- } \\
\text { nocimientos específicos } \\
\text { que brida el texto a la au- } \\
\text { diencia. }\end{array}$ & $\begin{array}{l}\text { Ensayos basados en los } \\
\text { requerimientos de las } \\
\text { otras asignaturas del cur- } \\
\text { so escolar y de obras lite- } \\
\text { rarias en estudio. }\end{array}$ \\
\hline $\begin{array}{l}\text { Cita sus escritos } \\
\text { cuando toma re- } \\
\text { ferencias. }\end{array}$ & $\begin{array}{lr}\text { Seleccionar } & y \\
\text { controlar un } \\
\text { tipo de texto en } \\
\text { sus componen- } \\
\text { tes globales. }\end{array}$ & $\begin{array}{l}\text { - Leer los productos genera- } \\
\text { dos por ély sus pares. } \\
\text { - Evaluación de los elementos } \\
\text { estructurales del texto. } \\
\text { - Identificación del requeri- } \\
\text { miento de escritura. } \\
\text { - Ajuste del documento pro- } \\
\text { ducido con base en la estruc- } \\
\text { tura. } \\
\text { - Revisión y aplicación de nor- } \\
\text { mas de escritura y de referen- } \\
\text { cia en el texto }\end{array}$ & $\begin{array}{l}\text { - Se cuestiona acerca de } \\
\text { lasposibles opciones que } \\
\text { permiten cumplir con las } \\
\text { demandas impuestas por } \\
\text { la tarea. } \\
\text { - Evoca información ne- } \\
\text { cesaria para llevar a cabo } \\
\text { la tarea. } \\
\text { - Identifica y corrige a } \\
\text { nivel de contenido ele- } \\
\text { mentos del escrito y es- } \\
\text { trategias utilizadas para } \\
\text { hacerlo más comprensi- } \\
\text { ble. }\end{array}$ & $\begin{array}{l}\text { Evaluación de escritos } \\
\text { con base en su estructura } \\
\text { y/o fin comunicativo. }\end{array}$ \\
\hline
\end{tabular}

Fuente: Elaboración propia 


\section{Los portafolios como instrumentos de evaluación}

Pozo define al portafolio como "Una carpeta de competencias o portafolios es una colección de documentos en diferentes soportes (cada vez más en soporte virtual) que describe y documenta el proceso de aprendizaje y las competencias de una persona, mostrando su desarrollo holístico y profesional a través de sus propias manifestaciones" (20I3:5). Desde esta definición, un portafolio para evaluar competencias comunicativas, recogerá las experiencias de escritura que los sujetos que aprenden experimentarán en el proceso de aprendizaje.

El portafolio como instrumento de evaluación de competencias no solo recogerá las experiencias exitosas de escritura sino también el proceso de corrección y mejoramiento que se va construyendo de manera sistémica con relación a los aprendizajes esperados en los estudiantes a nivel del currículo y de la competencia de escritura.

Según lo propone Moraine (20I4:98), con relación al proceso de construcción de portafolios evaluativos:

“desde la matriz de planificación de la evaluación, los procesos están orientados a cons-

truir desde las funciones ejecutivas superiores de los estudiantes; el uso de la vista previa y

de la revisión como estrategias de selección de evidencias que el estudiante consolidará en

su portafolio para lograr una visión más amplia de su capacidad de escribir”.

El portafolio entonces recogerá desde la tabla de especificaciones, los productos que se vayan construyendo de manera operativa, en cada uno de los bloques curriculares de la propuesta, permitiendo que la evaluación de la escritura, no solo considere a su objeto evaluable en sí, sino también los objetos de conocimiento prescritos en el currículo oficial de formación.

Debe ser claro, que el portafolio responderá a un pedido específico de evaluación, esto construido desde los intereses del sujeto, su propia evolución y el currículo oficial vigente para el curso. De esta forma, el portafolio que se utilizará para evaluar el aprendizaje de la competencia de escritura en segundo de bachillerato (grupo experimental de la investigación) constará con las siguientes evidencias: 
Tabla 4: Relación Bloque Curricular, Sistema de contenidos y Tarea de escritura

\begin{tabular}{|l|l|l|}
\hline \multicolumn{1}{|c|}{ Bloque Curricular } & \multicolumn{1}{|c|}{ Sistema de contenidos } & \multicolumn{1}{|c|}{$\begin{array}{c}\text { Tarrea de escriturara } \\
\text { requerida }\end{array}$} \\
\hline Lo abundante y lo fugaz & $\begin{array}{l}\text { Gérmenes de la Literatura Fantástica, Mágica y } \\
\text { Maravillosa en la descripción excesiva, exótica e } \\
\text { idealista de las Crónicas de Indias (Álvar Núñez } \\
\text { Cabeza de Vaca, Hernán Cortez, Cristóbal Co- } \\
\text { lón) }\end{array}$ & $\begin{array}{l}\text { Resumen de los aportes de las } \\
\text { Crónicas de Indias. }\end{array}$ \\
\hline Esclavitud y libertad & $\begin{array}{l}\text { Creaciones poéticas acerca de la libertad indivi- } \\
\text { dualy colectivay de denuncia social (Paz, Espron- } \\
\text { ceda, Clarín) } \\
\text { Características e influencia de los relatos cortos, } \\
\text { en las luchas libertarias (Montalvo, Echeverría, } \\
\text { Sarmiento). }\end{array}$ & $\begin{array}{l}\text { Comentario crítico sobre dos } \\
\text { de los autores analizados. }\end{array}$ \\
\hline Realidad y evasión & $\begin{array}{l}\text { El pasilloccuatoriano: fuentes deinspiración. Los } \\
\text { poetas “decapitados" ecuatorianos: principales } \\
\text { exponentes y sus obras (temas frecuentes, reali- } \\
\text { dad cotidiana, filosofía de vida) }\end{array}$ & $\begin{array}{l}\text { Ensayo crítico sobre la pro- } \\
\text { ducción de la generación de- } \\
\text { capitada ecuatoriana }\end{array}$ \\
\hline Lo individual y lo colectivo & $\begin{array}{l}\text { Recursos narrativos en la expresión de los } \\
\text { problemas sociales actuales y en la Generación } \\
\text { del Treinta. Semejanzas y diferencias. Principales } \\
\text { exponentes (Pareja Diezcanseco, Aguilera Malta, } \\
\text { De la Cuadra). }\end{array}$ & $\begin{array}{l}\text { Análisis comentado sobre la } \\
\text { producción literaria de la ge- } \\
\text { neración del 3o }\end{array}$ \\
\hline
\end{tabular}

Fuente: Elaboración propia

La recolección oportuna de estos elementos, asegura la práctica continua sugerida para la construcción de los indicadores de competencia (véase tabla 3) y garantiza la atención del currículo oficial del Ecuador. 


\section{Conclusiones}

- La evaluación de la competencia de escritura, estará enfocada desde las dimensiones lingüística y pragmática de la comunicación, de tal manera que desde la primera dimensión los sujetos desarrollarán la capacidad de comprender y utilizar el lenguaje y en la segunda dimensión la utilidad del mismo en los entornos de comprensión y comunicación.

- Para evaluar la competencia comunicativa de escritura, es necesario establecer indicadores propios y ajustados al entorno comunicativo así como a las demandas curriculares para poder determinar las tareas de evaluación, respondiendo al episteme de la evaluación auténtica, que sostiene que ésta debe ser lo más cercana a la realidad del estudiante.

- Los portafolios permiten evidenciar la capacidad metacognitiva de los sujetos que aprenden a escribir, recoge información final y de proceso, lo que permite una evaluación integral del uso de las capacidades adscritas a la competencia comunicativa en vía escrita en el nivel básico de desempeño, esto es, la expresión por escrito de sus ideas en textos cortos, resúmenes y ensayos escolares; recalcando que no es la agrupación arbitraria de evidencias, sino la selección minuciosa de productos específicos requeridos desde los intereses de los estudiantes y el diseño curricular base del país. 


\section{Referencias bibliográficas}

- Álvarez, I. (20I2). Aprendizaje estratégico y desarrollo profesional . España: FUNIBER.

- Chomsky, N. (1999). Elprograma minimalista. España: Editorial Alianza.

- Desinano, N., y Avendaño, F. (2006). Didáctica de las ciencias del lenguaje: Enseñar ciencias del lenguaje. Argentina: Editorial Homosapiens.

- Florez, R., Torado, C., Arévalo, I., Mesa, C., Modragon, S., y Pérez, C. (2005). Habilidades metalingüísticas, operaciones metacognitivas y su relación con los niveles de competencia en lectura y escritura: Un estudio Exploratorio. Forma y función, I5-44.

- Mendoza, A. (2006). Didáctica de la Lengua y la Literatura. España: Editorial Prentice Hall.

- Mendoza, F., Terranova, J., Zambrano, V., \& Macías, M. (3o de Marzo de 20I4). Estrategias de sensibilización y atención para la generación de interés en el aprendizaje de Lengua. International Journal of Develpomental and Educational Psychology, 2(I), I7-30.

- Ministerio de Educación. (2OI2). Estándares de calidad educativa. Quito, Ecuador: Autor.

- Moraine, P. (20I4). Las funciones ejecutivas del estudiante. Madrid: Editorial Narcea S.A.

- Niño, V. M., y Pachon, T. (2009). Cómo formar niños escritores: La estrategia del taller. Bogotá: ECOE Ediciones.

- Pérez, Á. (20I2). Educarse en al Era digital. Madrid: Editorial Morata.

- Pilleux, M. (200I). Competencia comunicativa y análisis del discurso. Estudios filológicos. Valdivia: Estudios pedagógicos, I43-I52.

- Pozo, J. Á. (20I3). Competencias profesionales: Herramientas de evaluación: el portafolios, la rúbrica y las pruebas situacionales (2a. ed.). Madrid: Editorial Narcea S.A.

- Tenbrick, T. (2006). Evaluación: Guía práctica para profesores. Madrid: Editorial Narcea.

- Trujillo, F. (2OIO). Objetivos en la enseñanza de lenguas extranjeras: De la competencia lingüística a la competencia intercultural. Ceusa, 35-46. 